\title{
Celastrol directly inhibits PFKM to induce weight loss and leptin
}

\section{sensitization}

\author{
Kang Wang ${ }^{1,2}$, Xiaobo $\mathrm{Wu}^{1,2}$, Yinghua Zhuang ${ }^{2}$, Huan $\mathrm{Sun}^{2}$, Fengchao Wang ${ }^{2^{\star}}$, Tao \\ Wang $^{2,3^{*}}$ and Zhiyuan Zhang ${ }^{2,3 *}$ \\ ${ }^{1}$ School of Life Sciences, Tsinghua University, Beijing 100084, China \\ ${ }^{2}$ National Institute of Biological Sciences, Beijing 102206, China \\ ${ }^{3}$ Tsinghua Institute of Multidisciplinary Biomedical Research, Tsinghua University, Beijing \\ 100084, China
}

*Correspondence wangfengchao@nibs.ac.cn (F. W.); wangta01006@nibs.ac.cn (T. W.); zhangzhiyuan@nibs.ac.cn (Z. Z.) 


\section{Abstract}

Despite the prevalence of obesity and related health consequences around the globe, effective treatments for inducing healthy weight loss are still lacking. Celastrol is a pentacyclic triterpene that was recently identified as a potent anti-obesity agent. Celastrol increases sensitivity to leptin, but the molecular target of celastrol is unknown. Therefore, the mechanisms by which this agent exerts its anti-obesity effect remain elusive. Using tissue-specific ABPP (activity-based protein profiling), we found that PFKM, a rate-limiting enzyme for glycolysis in skeletal muscle, is a direct target of celastrol. Celastrol inhibited PFKM enzymatic activity, and Pfkm knockout mice were resistant to a high fat diet, were hypersensitive to exogenous leptin, and were unresponsive to celastrol. PFKM inhibition led to activation of AMPK and inactivation of $A C C$ in cultured myotubes and mouse skeletal muscle. Specific loss of AMPK in muscle significantly attenuated the anti-obesity effects of celastrol. Further, PFKM inhibition and subsequent activation of the AMPK/ACC signaling pathway reduced levels of free fatty acids by switching energy expenditure and consequently decreasing levels of SOCS1 expression, which are both required for leptin sensitization in 293t/hLepRb cells and mice. Finally, using a high throughput compound screen we identified an alternative PFKM inhibitor, 3-79, which exhibits a strong anti-obesity effect and non-covalent binding capacity. This compound is a promising agent for treating obesity in the clinic. 


\section{Introduction}

Obesity is a metabolic disorder involving abnormal or excessive fat accumulation, affecting more than 900 million people worldwide 1 . Obesity is becoming one of the major risk factors for a series of human diseases, including type 2 diabetes, cardiovascular disease, musculoskeletal disorders, and cancer. The adipocyte-derived hormone leptin functions as an anorexic hormone, regulating energy homeostasis, metabolism, and neuroendocrine function ${ }^{2-4}$. Levels of leptin therefore provide information about peripheral energy conditions. Leptin reduces body weight primarily by stimulating anorexigenic neuropeptides including POMC (proopiomelanocortin) and CART (cocaine- and amphetamine-regulated transcript), and by repressing orexigenic neuropeptides such as AgRP (agouti-related protein) and NPY (neuropeptide Y) in the CNS (central nervous system $)^{5-8}$. Leptin can also directly increase catabolism of peripheral tissues ${ }^{9-11}$. Although leptin reduces food intake and body weight in lean mice, high levels of leptin fail to suppress food intake and stimulate energy expenditure in mouse models of DIO (diet-induced obesity) and in obese humans ${ }^{2,12,13}$. Because obese individuals develop resistance to leptin, leptin-based clinical therapies for treating DIO are not effective.

Through an in silico drug screen, Ozcan and his co-workers identified celastrol as a potential leptin sensitizer and anti-obesity agent. Testing in DIO mice revealed that celastrol suppresses food intake, increases energy expenditure, and reduces body weight $^{14}$. It was later found that celastrol induces the expression of Interleukin 1 receptor 1 (IL1R1) in the hypothalamus, and that this mediates celastrol's leptin sensitization and anti-obesity effects ${ }^{15}$. However, there is no evidence that celastrol directly binds IL1R1. 
Several putative targets of celastrol have been identified, including IkB kinase, Hsp90/CDC37, protein tyrosine phosphatase 1B (PTP1B), T-cell PTP (TCPTP), and the proteasome, but none of these mediate the anti-obesity effects ${ }^{16-20}$. Thus, the direct molecular targets by which celastrol regulates leptin sensitivity remain elusive.

Skeletal muscles of obese individuals exhibit dramatic increases in glycolytic rate and lipid content 21,22 . In addition, activity of PFK (6-phosphofructokinase), a rate-determining enzyme in glycolysis, in skeletal muscle is proportional to alterations in body weight21,23. There are three isoforms of PFK, namely the muscle type (PFKM), liver type (PFKL) and platelet type (PFKC). While most tissues express all three isoforms, skeletal muscles express only PFKM²4.

Using ABPP (activity-based protein profiling), we identified PFKM as a direct target of celastrol. Celastrol inhibits PFKM, leading to activation of AMPK (Adenosine 5'-monophosphate-activated protein kinase), leptin sensitization, and anti-obesity effects. We further demonstrate that celastrol switches energy demand from glycolysis to free fatty acid oxidation in skeletal muscle, and that this metabolic change in skeletal muscle elevates both cellular and circulatory fatty acid levels. This systematically increases leptin sensitivity and protects animals from DIO.

\section{Results}

To identify targets of celastrol that lead to leptin sensitivity, we utilized activity-based protein profiling (ABPP). We designed a celastrol-based probe, 1-85, and added a propargyl tag to the $\mathrm{C}_{28}$ of celastrol to click to a biotin tag for further enrichment (Figure 
1A). We first tested the anti-obesity effects of 1-85 in mouse models of DIO using celastrol as a positive control. DIO mice treated with celastrol or 1-85 for 20 days exhibited reduced body weight compared to controls (Figures S1A and S1B). Upon co-treatment with leptin, both celastrol and 1-85 reduced body weight in 8 week-old mice fed with normal chew diet compared to treatment with leptin or celastrol/1-85 alone (Figure S1C). Importantly, these results demonstrated that the anti-obesity effects of celastrol are conserved in this probe, providing an opportunity to identify the desired target.

Because the fundamental cause of obesity is imbalance between energy intake and expenditure, we performed ABPP using 1-85 as probe in six organs, namely muscle, liver, intestine, adipose, stomach, and brain (Figure 1B). Significant differences in protein bands between samples treated with probe vs. controls (samples treated with probe plus 10 -fold of celastrol as a probe antagonist) were observed only in skeletal muscle (Figure 1C). Protein bands for both control and probe skeletal muscles were cut out and analyzed by LC-MS/MS, identifying a series of candidate proteins involved in metabolism (Table S1). Among these candidates was PFKM (Phosphofructokinase, Muscle), one of the three rate-determining enzymes in glycolysis. PFKM was one of the most enriched proteins and we confirmed this enrichment via western blot (Figure 1D). To determine direct effects of celastrol on enzymatic activity of PFKM, we ectopically expressed and purified the recombinant human PFKM in 293 T cells, and found that both celastrol and 1-85 potently inhibited PFKM activity with $\mathrm{IC}_{50}$ values of 349 and $507 \mathrm{nM}$, respectively. This indicates that PFKM is a true target of celastrol (Figure 1E). We next performed seahorse glycolysis stress test using mature L6 myotube cells derived from skeletal muscle to examine if 
celastrol could reduce glycolysis by inhibiting PFKM in vivo. Both celastrol and shRNA (short-hairpin RNA) of Pfkm did not alter basal glycolytic rates measured by extracellular acidification rate (ECAR) (Figures $1 \mathrm{~F}, 1 \mathrm{l}$ and $\mathrm{S} 2$ ), whereas adding oligomycin to block mitochondrial respiration rapidly increased the glycolytic rate (ECAR rate, $47.12 \pm 3.56$ $\mathrm{mpH} / \mathrm{min}$ ) in control cells. Treatment with celastrol (ECAR rate, $30.58 \pm 6.43 \mathrm{mpH} / \mathrm{min}$ ) and Pfkm shRNA (ECAR rate, $32.96 \pm 3.10 \mathrm{mpH} / \mathrm{min}$ ) reduced this increase in glycolysis (Figures $1 \mathrm{~F}$ and $1 \mathrm{H}$ ). These data demonstrated that inhibition of PFKM by celastrol in L6 cell reduced the overall glycolytic capacity without affecting the basal glycolytic rate, suggesting that celastrol may only inhibit glycolysis in skeletal muscle when glycolytic rates are high. This could explained the previous results that celastrol reduced body weight only in DIO or leptin administered mice, since it has been demonstrated that obesity and leptin treatment increases glycolysis rate in skeletal muscle3,21,23.

To verify that PFKM is a bona fide target of celastrol in mediating weight loss, we generated Pfkm knockout (Pfkm KO) mice. Considering that PFKM deficiency might cause embryonic lethality, we attempted to generate Pfkm conditional knockout mice using Cre-loxP system (Pfkm KO, See method and Figure S3). However, homozygous Pfkm KO mice totally lacked PFKM expression in skeletal muscle, liver, and hypothalamus without Cre recombinase expression, which might because that the loxP insertions disrupt the transcription of $P f k m$ (See method and Figure 1J). Although Pfkm KO mice showed no obvious differences in behavior or development compared to wild-type littermates, Pfkm KO mice gained weight much more slowly than controls when fed a HFD (high fat diet). In addition, serum leptin levels of Pfkm KO mice were lower than 
their wild-type littermates (Figures $1 \mathrm{~K}$ and S4A). After 20 weeks of HFD feeding, both Pfkm KO and wild-type mice were subjected to intraperitoneal administration of celastrol for 20 days. While wild-type mice lost body weight upon treatment with celastrol, body weight of Pfkm KO mice was unaffected, indicating that Pfkm mutant mice failed to response to celastrol (Figures $1 \mathrm{~L}$ and $1 \mathrm{M}$ ). To investigate if PFKM inhibition mediates the effects of celastrol on leptin sensitization, we treated both 8-week-old wild-type and Pfkm KO mice with leptin with or without celastrol. While wild-type mice treated with leptin showed mild effects in body weight, Pfkm KO mice showed significant reductions in body weight compared to wild-type controls. However, wild-type mice exhibited dramatic reductions in body weight upon co-injection of celastrol and leptin. By contrast, celastrol did not further reduce the body weight of leptin treated Pfkm KO mice (Figure 10). These data indicated that Pfkm KO mice are more sensitive to leptin, and support that PFKM is a direct target of celastrol in promoting weight loss.

Considering that PFKM is a key regulator of glycolysis, pharmacological inhibition may reduce the contribution of energy from glycolysis. This would force cells to utilize other energy-generating pathways and thus exert profound influences on metabolic networks ${ }^{25}$. We then performed an unbiased, untargeted metabolomics analysis of an L6 cell line treated with celastrol (Table S2). Although ATP levels were unchanged, both ADP and AMP levels increased significantly after celastrol treatment (Figure 2A). In addition, malonyl-CoA levels were decreased (Figure 2A). Elevated levels of ADP and AMP could potentially activate AMPK (Adenosine 5'-monophosphate-activated protein kinase), which inhibits ACC (Acetyl-CoA carboxylase) catalyzing carboxylation of acetyl-CoA to produce 
malonyl-CoA. Therefore, we checked the activated states of AMPK, and found that celastrol treatment activated AMPK (phosphorylation of AMPK on thr172 is an activated form) and inactivated ACC (phosphorylation of ACC on ser790 is an inactivated form) in the L6 cell line (Figure 2B). AICAR (an AMPK activator) or acute glucose starvation had similar effects on the AMPKIACC pathway. Consistent with a reduction in malonyl-CoA, which is a key intermediate in fatty acid synthesis and inhibits the beta-oxidation of fatty acids, the concentration of FFA (free fatty acids) was lower in L6 cells treated with celastrol (Figure 2C and table S3). We next verified that celastrol activated AMPK in vivo. Celastrol treatment of wild-type DIO mice significantly increased phosphorylation forms of AMPKThr172 and ACCSer79 in skeletal muscle but not in liver or hypothalamus (Figure 2F, $2 \mathrm{G}$ and $2 \mathrm{H}$ ). Moreover, phosphorylation of AMPK was increased in muscle of Pfkm KO mice raised on HFD; this was not further increased by celastrol (Figures $1 \mathrm{~N}, \mathrm{~S} 5 \mathrm{~A}$ and S5B). To investigate if the AMPK/ACC pathway in skeletal muscle mediates the effects of celastrol on weight loss, we specifically knocked out the a subunit of AMPK in skeletal muscle of $A M P K \alpha 1 \alpha 2^{10 x p / / 10 x p} ; C K M-C r e E R(A M P K \alpha 1 \alpha 2$ mKO) mice. When fed a HFD for 20 weeks, AMPKa1a2 mKO mice had same weight as controls. Leptin level in DIO AMPK a1a2 mKO mice were equivalent to wild-type (Figure S4B). Importantly, loss of AMPK in muscles significantly attenuated the weight-loss effects of celastrol (Figures 2D and 2E). These results suggest that activation of AMPK is required for the weight-loss effects of celastrol.

We next examined whether celastrol-induced leptin sensitization is also mediated by the activation of AMPK. STAT3 activation (phosphorylation of STAT3 on Tyr705) is one of 
the most important downstream targets of leptin26, and we observed robust phosphorylation of STAT3 after acute intraperitoneal leptin administration in both the hypothalamus and skeletal muscle (Figure S6). We therefore used levels of STAT3Tyr705 phosphorylation as an indicator of leptin sensitivity in all tissues we investigated. Celastrol treatment increased STAT3 ${ }^{\text {Tyr705 }}$ phosphorylation in all tissues examined including liver, hypothalamus, and skeletal muscles of wild-type DIO mice, despite the fact that activation of AMPK was only detected in muscles. In contrast, levels of STAT3Tyr705 phosphorylation were higher in muscles of Pfkm KO mice. This was not further increased by celastrol (Figure $1 \mathrm{~N}$ ). Moreover, muscle-specific knockout of $A M P K \alpha 1 \alpha 2$ attenuated the ability of celastrol to induced STAT3Tyr705 phosphorylation in all these tissues (Figures 2F-2H).

Since inhibiting PFKM in muscles via celastrol increased cellular responses to leptin in most tissue, changes in circulating factors may mediate this systemic leptin sensitization. Previous research has demonstrated that obesity is associated with elevated levels of circulating fatty acids ${ }^{27}$. Based on the dramatic reductions in FFA in L6 cell after celastrol treatment, we assumed that high levels of FFA may interfere with the leptin signaling pathway. We then used a previously reported leptin-sensitive 293T cell model (293T/hLepRb) in which the human signaling form of the leptin receptor (hLepRb) is stably transfected ${ }^{28}$. We observed that extremely high levels of celastrol could inhibit both PFKM and PFKL $\left(\mathrm{IC}_{50}=916.40 \pm 35.66 \mathrm{nM}\right)$, the dominant isoform of phosphofructokinase in 293T cell (Figure S7A). Celastrol treatment inhibited glycolytic capacity and reserve of $293 \mathrm{~T} / \mathrm{hLepRb}$ cells in seahorse glycolysis stress assay (Figure S7B). Treatment of $293 \mathrm{~T} / \mathrm{hLepRb}$ cells with $10 \mathrm{ng} / \mathrm{ml}$ leptin was sufficient to induce 
phosphorylation of STAT3Tyr705, and pretreating cells with palmitic acid (PA) attenuated leptin-induced STAT3 phosphorylation in a dose dependent manner (Figure 3A). Importantly, treatment with celastrol or AICAR, as well as glucose starvation, dramatically counteracted the suppressing effects of PA on leptin-induced phosphorylation of STAT3. Moreover, etomoxir, which reduces fatty acid oxidation by inhibiting carnitine palmitoyltransferase-1 (CPT-1), abolished the effects of celastrol, AICAR, or glucose starvation on leptin signaling (Figure 3B and $3 \mathrm{C}$ ). These results, together with the fact that celastrol promotes consumption of FFA, indicate that celastrol sensitizes leptin signaling by reducing cellular FFA.

The SOCS family of proteins play important roles in the negative regulation of leptin signaling. We therefore reasoned that PA treatment could increase expression of SOCS1 and/or SOCS3. Since SOCS3 mRNA was not detected in 293T/hLepRb cells (data not show), we only measured the expression of SOCS1. SOCS1 was expressed more than 4-fold higher after 293T/hLepRb cells were treated with PA, and co-treatment with AICAR or glucose starvation reduced the expression of SOCS1 to levels without PA. Similarly, celastrol counteract the ability of PA to reduce expression of SOCS1 in a dose dependent manner (Figure 3D), suggesting that SOCS1 is a key regulator of PA-induced leptin desensitization. We next knocked down SOCS1 in 293T/hLepRb cells by shRNA, and found that these cells maintained phosphorylation of STAT3 upon PA treatment (Figures 3E and S8). We next verified these finding in DIO mice models, and found that total levels of circulating FFAs and most individual FFA species were largely decreased in wild-type mice following treatment of celastrol or Pfkm KO mice. By contrast, administration of 
celastrol to Pfkm KO mice showed no further reduction effects (Figure 3F and Table S4). Celastrol treatment significantly reduced SOCS1 mRNA levels in muscles, while muscle-specific $A M P K$ knock-out attenuated the effect of celastrol on SOCS1 mRNA levels (Figure 3G). These results suggest that a reduction in circulatory FFAs mediates the effects of celastrol on leptin sensitization.

The anti-obesity effects of celastrol (by inhibiting PFKM in muscles), together with previous studies demonstrating that celastrol has excellent pharmacokinetic properties, indicate that celastrol may be a potent anti-obesity drug 29 . However, celastrol covalently binds to multiple targets leading to adverse side effects, thereby weakening the potential usage of celastrol as an anti-obesity drug ${ }^{30,31}$. Because celastrol is a complicated natural product, its pharmaceutical properties are difficult to improve. We therefore screened for alternative PFKM inhibitors from $\sim 6000$ pilot compounds (methods and supporting info). The twelve most potential compounds were selected for further analysis (Table S5). We measured the $\mathrm{IC}_{50}$ of these twelve compounds to PFKM in buffers with either $0.5 \mathrm{mg} / \mathrm{ml}$ bull serum albumin or $1 \mathrm{mM}$ glutathione to exclude nonspecific protein binding and oxidizing, respectively. One of compounds, 3-79, inhibited PFKM in both systems (Figures 4A, 4B and Table S5). Seahorse glycolysis stress test revealed that $1 \mu \mathrm{M}$ 3-79 reduced glycolytic capacity and reserve with minimal effects on the basal glycolytic rate (Figures $4 \mathrm{C}-4 \mathrm{~F}$ ) in $\mathrm{L6}$ cells (as seen with celastrol). Moreover, 3-79 increased phosphorylation of AMPKThr172 and ACCSer79 in both L6 cells and skeletal muscles of DIO mice, and boosted leptin response in muscles (Figures $4 \mathrm{G}$ and $4 \mathrm{H}$ ). Since 3-79 is an alternative inhibitor of PFKM, we next asked if 3-79 could induce anti-obesity effects in animal models. 
Intraperitoneal injection of 3-79 gradually reduced the body weight of DIO mice at comparable level as celastrol (Figures $4 \mathrm{I}$ and $4 \mathrm{~J}$ ). As with celastrol, injection of 3-79 at higher concentration did not have obvious effects on regular lean mice, indicating minimal toxicity effects of 3-79 (Figure S9). Considering that 3-79 has no covalent binding capacity and modification potential, it represents a potential PFKM inhibitor that should be further optimized.

\section{Discussion}

The discovery that leptin regulates food intake and energy expenditure heightened our understanding of bodyweight regulation. However, the attempt to cure obesity with leptin failed in most obese individuals that were not genetically deficient in leptin signaling. Circulating levels of leptin were extremely high in these obese patients, and the addition of exogenous leptin failed to decrease appetite and increase energy expenditure ${ }^{12,13}$. The cause of this leptin resistance has not been fully illuminated, but inflammation ${ }^{32,33}$, ER (endoplasmic reticulum) stress ${ }^{34}$, and autophagy ${ }^{35}$ are involved in obesity-induced leptin resistance. The discovery that celastrol can induce leptin sensitization, thereby reducing food intake and body weight in DIO mice with little effect on lean animals, re-established hope that leptin-oriented strategies could be used to treat obesity ${ }^{14}$. Celastrol treatment led to a significant decrease in PERK phosphorylation, but it fails to increase ER stress genes involved in hypothalamic ER stress, indicating that celastrol may not be directly involved in ER stress signaling20. Using a celastrol-based ABPP probe, named 1-85, we revealed that PFKM is a target of celastrol in skeletal muscle. Celastrol inhibited PFKM 
activity in vitro, and thus reduced glycolytic capacity in skeletal muscle-derived L6 myotube cells. Pfkm KO mice did not gain weight when fed a HFD, and did not lose weight in response to celastrol treatment. Moreover, the alternative PFKM inhibitor, 3-79, exhibited anti-obesity effects that mimicked celastrol. Altogether, PFKM is a major target of celastrol in promoting weight loss.

Leptin is a key regulator of fatty acid metabolism in skeletal muscle tissues, as this hormone stimulates fatty acid oxidation in muscle, primarily by activating the AMPK/ACC axis $^{36,37}$. High level of glycolysis have been observed in both obese individuals and animals with leptin treatment 3,21 . As a rate-limiting enzyme in glycolysis, the activity of PFK is positively correlated with body weight gain ${ }^{23}$. As an inhibitor of glycolysis, celastrol increased cellular AMP levels, activating AMPK and inactivating ACC by phosphorylating ACCSer79 in L6 cells, thereby promoting fatty acid oxidation. Therefore, celastrol-treated skeletal muscle cells produced more ATP from fatty acid oxidation rather than glycolysis. Similarly, Pfkm KO mice increased phosphorylation of AMPK Thr172 when raised on a HFD, and were resistant to celastrol. Moreover, specific knock-out of $A M P K$ in skeletal muscle largely attenuated the anti-obesity effects of celastrol, supporting the notion that activation of AMPK in skeletal muscle is a key step in anti-obesity effects of celastrol.

As a result of increased fatty acid oxidation and decreased glycolysis, FFA levels were reduced dramatically in L6 cells upon celastrol treatment. Importantly, circulatory FFAs were decreased in DIO mice treated with celastrol or in Pfkm KO mice. In obese animals, fatty acid oxidation levels were decreased and glycolysis levels were increased in skeletal muscle, which elevated circulating FFAs. Using the 293t/hLepRb model, 
treating cells with palmitic acid significantly attenuated cellular leptin response. Moreover, boosting cellular fatty acid oxidation by AICAR treatment or glucose starvation abolished this PA-induced leptin desensitization, whereas inhibiting CPT-1 by etomoxir abolished the effects of AICAR and glucose starvation on leptin signaling. However, inhibition of CPT1 in hypothalamus successfully suppresses food intake ${ }^{38}$. Since hypothalamus only uses glucose and ketone as energy sources, inhibition of CPT1 might not reduce FFAs, but rather sense circulating FFAs. In summary, our work supports that a functional metabolic state is necessary for leptin signal transduction, and that celastrol promotes systematic sensitization to leptin signaling by reducing circulatory FFAs.

The SOCS family of proteins has long been considered key negative regulators of leptin signal transduction. Besides SOCS3, SOCS1 also binds and inhibits the JAK family 39,40 , and the expression of SOcS1 is increased in skeletal muscle and the hypothalamus of obese animals ${ }^{41,42}$. However, it has been shown that leptin administration induces SOCS3 but not SOCS1 in the hypothalamus ${ }^{43}$. Our work demonstrated that the metabolic state could influence leptin signal transduction by regulating SOCS1 expression in both 293t/hLepRb cells and mouse skeletal muscles. In 293t/hLepRb cells, in correlation with diminishing leptin signaling, PA treatment reduced SOCS1 expression, and knocking down SOCS1 abolished PA-induced leptin desensitization. Moreover, celastrol treatment largely reduced SOCS1 mRNA levels in wild-type but not in AMPK mKO skeletal muscles. Therefore, induction of socs1 expression by circulatory FFAs is involved in obesity-associated systemic leptin resistance. Since both IL1R1 and SOCS1 play important roles in leptin sensitization and 
inflammation ${ }^{15} 44$, crosstalk between these two processes may be an important issue to be investigated.

PFKM is the only phosphofructokinase in muscle. In contrast, PFKM is expressed in the brain and most other tissues express PFKL and PFKC as well, forming homo- and hetero-tetramers ${ }^{24}$. It has been reported that mice lacking PFKM in the brain but not muscles have greatly reduced fat stores under chew diet ${ }^{45}$. This work indicated a close relationship between activity of central PFKM and bodyweight regulation. However, the effects of peripheral PFKM on body weight regulation and whether these effects still maintained in obesity models have not been clarified. In Pfkm KO mice we generated (complete loss of PFKM), no obvious differences in behavior and development were observed in comparisons to wild-type littermates, except for anti-obesity effects when fed a HFD. Importantly, Pfkm KO mice are completely resistant to celastrol. In contrast, specifically disrupting AMPK in muscles attenuated the anti-obesity effects of celastrol, and weakened celastrol-induced leptin sensitization in liver, hypothalamus, and skeletal muscles. Considering that skeletal muscle accounts for $40 \%$ of bodyweight and consumes more than $30 \%$ of oxygen in the rest state ${ }^{46}$, metabolic switching in skeletal muscle could systematically improve leptin signal transduction in multiple tissues through metabolites like FFAs.

As the most potent anti-obesity agent, celastrol is a promising drug candidate for treating obesity in humans. However, celastrol is a triterpene that contains an electrophilic quinone methide moiety, which enables celastrol to covalently bind to the cysteine of target proteins. Moreover, as a complicated nature product, the pharmaceutical properties 
of celastrol are difficult to improve by modifying the compound. Thus, despite the dramatic leptin sensitizing effects, the used of celastrol as an anti-obesity drug is severely limited by resulting adverse side effects ${ }^{30,31}$. Here we discovered that celastrol's anti-obesity function depends on its ability to inhibit PFKM. Based on key anti-obesity effects on targeting PFKM, we identify a lead compound, 3-79, which efficiently inhibited PFKM activity. Importantly 3-79 reduced body weight of DIO mice as efficiently as celastrol with minimal side-effects. All of this indicated inhibition of PFKM by small molecular holds great promise as a future therapeutic intervention to cure obesity.

\section{Acknowledgments}

We thank the Metabolomics Center, Proteomics Facility and Chemistry Center at the National Institute of Biological Sciences, Facility Center of Metabolomics and Lipidomics at Tsinghua University and Beijing Omics biological technology Co., Ltd. for technical assistance. We thank Dr. Li Li and Dr. Dapeng Ju for helpful discussions. We thank Dr. D. O'Keefe for helpful discussions and comments on the manuscript. This work was supported by grants from the National Natural Science Foundation of China (81670891 and 81870693) awarded to T. Wang, and National Major Scientific and Technological Special Project for "Significant New Drug Development” during the Twelfth Five-year Plan Period (2013ZX0950910) awarded to Z. Zhang.

\section{Competing interests}

The authors declare no competing financial interests. 
bioRxiv preprint doi: https://doi.org/10.1101/2020.09.06.284752; this version posted September 8, 2020. The copyright holder for this preprint (which was not certified by peer review) is the author/funder. All rights reserved. No reuse allowed without permission. 


\section{Figure Legends}

Figure 1. Celastrol causes weight loss by inhibiting PFKM. (A) Chemical structures of celastrol and 1-85. (B) Schematic diagram of ABPP. A 10-fold excess of celastrol was added to antagonize the 1-85 probe in controls. (C) The 1-85-bound proteins were analyzed by SDS-PAGE followed by silver staining. ABPP experiments were performed using homogenates of six organs: muscle, liver, intestine, adipose, stomach, and brain. (D) Western blot of 1-85-bound proteins in homogenates of skeletal muscle from 8-week-old lean mice using anti-PFKM antibodies. $(E)$ The $\mathrm{IC}_{50}$ of celastrol and 1-85 was measured using recombinant PFKM. (F) Seahorse glycolysis stress test of L6 cells treated with celastrol or PFKM shRNA. (G-I) The glycolysis capacity $(\mathrm{G})$, glycolytic reverse $(\mathrm{H})$, and basic glycolytic rate $(\mathrm{I})$ of each group in $(\mathrm{F})$. (J) PFKM expression levels in muscle, liver, and hypothalamus from wild-type DIO mice, wild-type lean mice, and Pfkm KO mice. HSP90 was used as an internal control. (K) Body weight of wild-type and Pfkm KO mice fed a HFD. Mice were fed a HFD right after weaning for 20 weeks. Body weight of wild-type and Pfkm KO mice increased by 1.9 -fold (from $14.16 \pm 2.47 \mathrm{~g}$ to $41.82 \pm 7.52 \mathrm{~g}$; $\mathrm{n}=11$ ) and 1.4-fold (from $12.55 \pm 1.94 \mathrm{~g}$ to $30.48 \pm 0.62 \mathrm{~g} ; \mathrm{n}=4$ ), respectively. (L-M) Celastrol failed to reduce body weight of Pfkm KO DIO mice. Wild-type and Pfkm KO mice were fed HFD for 20 weeks to induce DIO, followed by daily intraperitoneal injection of celastrol $(0.15 \mathrm{mg} / \mathrm{kg})$ for 20 days. The time course of body-weight changes $(\mathrm{L})$ and total body-weight changes (M) are shown. Celastrol reduced body weight of wild-type mice after 20-day treatment (from $45.8 \pm 2.10 \mathrm{~g}$ to $40.27 \pm 3.56 \mathrm{~g}, \mathrm{n}=3$ ), compared to vehicle treatment (from $40 \pm 1.57 \mathrm{~g}$ to $45.53 \pm 2.54 \mathrm{~g}, \mathrm{n}=3$ ). Neither vehicle (from $29.00 \pm 1.73 \mathrm{~g}$ to $31.2 \pm 2.98 \mathrm{~g}, \mathrm{n}=3$ ) nor celastrol $(0.15 \mathrm{mg} / \mathrm{kg}$ ) (from $29.15 \pm 2.36 \mathrm{~g}$ to $29.50 \pm 1.67 \mathrm{~g}, \mathrm{n}=4$ ) treatment reduced the body weight of Pfkm KO mice. (N) Western blot indicated that Pfkm $\mathrm{KO}$ and celastrol treatment induced phosphorylation of AMPK ${ }^{\text {Thr172 }}$ and $\mathrm{ACC}^{\mathrm{Ser} 79}$ in skeletal muscles of DIO mice. (O) Pfkm KO mice were sensitive to leptin, but failed to further response to celastrol. Eight-week-old wild-type and Pfkm KO lean mice were intraperitoneally injected with either vehicle or celastrol $(0.15 \mathrm{mg} / \mathrm{kg})$ for four days. On the third and fourth days, mice were treated with either saline or leptin $(5 \mathrm{mg} / \mathrm{kg})$. Body-weight 
changes of WT control $(-0.24 \pm 0.49 \mathrm{~g} ; \mathrm{n}=8), \mathrm{WT}+\mathrm{L}(-0.40 \pm 0.29 \mathrm{~g} ; \mathrm{n}=10)$, Pfkm KO+L $(-0.95 \pm 0.10 \mathrm{~g} ; \mathrm{n}=4), \mathrm{WT}+\mathrm{Cel}+\mathrm{L}(-0.975 \pm 0.40 \mathrm{~g} ; \mathrm{n}=4)$ and Pfkm KO+Cel+L $(-1.08 \pm 0.19 \mathrm{~g}$; $\mathrm{n}=4$ ) between day 5 and day 3 were calculated.

Figure 2. Celastrol reduces body weight and elevates systemic leptin response by activating AMPK in skeletal muscles. (A) The changes in ATP (from $100 \pm 55.51 \%$ to $73.19 \pm 60.46 \%$ ), ADP (from $100 \pm 11.74 \%$ to $192.65 \pm 46.53 \%$ ), AMP (from $100 \pm 10.63 \%$ to $247.09 \pm 55.43 \%$ ), and Malonyl-CoA (from $100 \pm 38.49 \%$ to $39.54 \pm 14.15 \%$ ) levels in L6 cell after $4 \mu \mathrm{M}$ celastrol treatment for $30 \mathrm{~min}(\mathrm{n}=3)$. (B) Western blot of $L 6$ cells treated with different concentrations of celastrol for $30 \mathrm{~min}$ or $1 \mathrm{mM} \mathrm{AICAR}$ or glucose starvation for 8 h. (C) Heatmap visualization of relative free fatty acid levels in L6 cells after treatment with $2 \mu \mathrm{M}$ or $4 \mu \mathrm{M}$ celastrol for $30 \mathrm{~min}$. The detailed data listed in table S3. ( $\mathrm{n}=3$ for each group) (D-E) Time course of percentage of body-weight changes (\%) during compound treatment

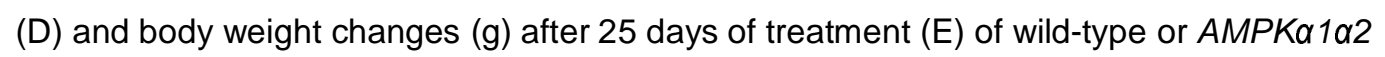
mKO DIO mice ( $n=4$ for vehicle treated wild-type mice, $n=5$ for $0.15 \mathrm{mg} / \mathrm{kg}$ celastrol treated wild-type or AMPKa1a2 mKO mice). (F-H) Western blot shows that celastrol induced phosphorylation of STAT3 ${ }^{\text {Tyr705 }}$ in skeletal muscles $(F)$, liver $(G)$, and hypothalamus $(\mathrm{H})$, and $A M P K \alpha 1 \alpha 2 \mathrm{mKO}$ attenuated the effects of celastrol. Both wild-type and AMPKa1 $12 \mathrm{mKO}$ DIO mice were intraperitoneally injected with celastrol $(0.15 \mathrm{mg} / \mathrm{kg})$ for 25 days.

Figure 3. Reduction of FFA by celastrol is involved in leptin sensitization. (A) Western blot analysis shows PA attenuated the response of $293 \mathrm{~T} / \mathrm{hLepRb}$ cells to leptin. The 293T/hLepRb cells were pretreated with different concentration of PA for $8 \mathrm{~h}$, followed by treated with $10 \mathrm{ng} / \mathrm{ml}$ leptin for $1 \mathrm{~h}$. (B-C) Celastrol suppressed the inhibitory effects of PA on leptin response. The 293T/hLepRb cells were pretreated with $2 \mu \mathrm{M}$ celastrol or 10 mM AICAR, or deprived of glucose for $8 \mathrm{~h}$, followed by treatment with $500 \mu \mathrm{M}$ PA for $8 \mathrm{~h}$ and subsequent treatment with $10 \mathrm{ng} / \mathrm{ml}$ leptin for $1 \mathrm{~h}$. (C) Quantification of relative pSTAT3 $^{\text {Tyr705 }}$ levels. $\beta$-Tubulin served as internal control (n=3). (D) QPCR was used to measure relative SOCS1 expressing levels in 293T/hLepRb cells co-treated with celastrol or AICAR or glucose starvation and $500 \mu \mathrm{M}$ PA $(n=3)$. (E) Western blot shows that 
293T/hLepRb cells with SOCS1 knocked-down maintained leptin response in the presence of PA. The 293T/hLepRb cells were transfected with SOCS1 shRNA expressing vector for $36 \mathrm{~h}$, followed by treatment with $500 \mu \mathrm{M}$ PA for $8 \mathrm{~h}$ and subsequent treatment with $10 \mathrm{ng} / \mathrm{ml}$ leptin for $1 \mathrm{~h}$. (F) Lipidomic analysis of FFA changes in serum of WT and Pfkm KO mice intraperitoneal treated with celastrol or vehicle for 20 days $(\mathrm{n}=4$ for $P f k m$ $\mathrm{KO}$ mice+celastrol, $\mathrm{n}=3$ for the other groups). The numerical data is listed in Table S4. (F) SOCS1 expression levels in muscles of wild-type or AMPKa1a2 mKO DIO mice. Both the wild-type and AMPKa 1a2 mKO mice were fed HFD for 20 weeks to induce DIO, followed by intraperitoneal injection of celastrol $(0.15 \mathrm{mg} / \mathrm{kg})$ for 20 days.

Figure 4. Identification of 3-79, a new PFKM inhibitor, as a potent candidate for pharmaceutic application. (A) Chemical structural of 3-79. (B) The $\mathrm{IC}_{50}$ of 3-79 on recombinant PFKM. (C-F) Seahorse glycolysis stress test shows that both glycolytic capacity (D, DMSO, $36.65 \pm 3.00 \mathrm{mpH} / \mathrm{min} \mathrm{n}=5$; 3-79, $9.40 \pm 4.11 \mathrm{mpH} / \mathrm{min}, \mathrm{n}=6$ ) and glycolytic reserve (E, DMSO, $24.80 \pm 5.22 \mathrm{mpH} / \mathrm{min} \mathrm{n}=5$; 3-79, $-0.04 \pm 1.52 \mathrm{mpH} / \mathrm{min}, \mathrm{n}=6$ ) were reduced by 3-79 treatment. The ECAR of basal glycolysis (F, DMSO, 11.85olysismpH/min $n=5 ; 3-79,9.44 \pm 3.96 \mathrm{mpH} / \mathrm{min}, \mathrm{n}=6$ ) remained unchanged. $(\mathrm{G}-\mathrm{H})$ Western blot analysis of $L 6$ cells $(G)$ and skeletal muscle of DIO mice $(H)$ reveal that 3-79 increased phosphorylation of AMPK ${ }^{\text {Thr172 }}$, ACC $^{\text {Ser79 }}$ and STAT3 ${ }^{\text {Tyr705 }}$. L6 cells were treated with different concentrations of 3-79 or $1 \mathrm{mM} \mathrm{AICAR}$ for $30 \mathrm{~min}$. The wild-type DIO mice were intraperitoneally injected with $3-79(0.5 \mathrm{mg} / \mathrm{kg})$ twice a day for 15 days. (I-J) 3-79 reduced body weight of wild-type DIO mice. Wild-type mice fed HFD for 20 weeks to induce DIO, followed by twice a day intraperitoneal injection of vehicle or 3-79 $(0.5 \mathrm{mg} / \mathrm{kg})$ twice a day for 15 days. The time course of relative body-weight changes $(I, n=5)$ and body-weight changes after a 15-day period of 3-79 treatment ( $\mathrm{J}$, vehicle, Day1 41.63 \pm 1.51 g vs. Day $1541.18 \pm 2.17 \mathrm{~g}$; 3-79, Day1 $41.66 \pm 1.79 \mathrm{~g}$ vs. Day15 36.82 $\pm 2.26 \mathrm{~g}$ ) are shown.

\section{References}

1. Di Cesare, M. et al. Trends in adult body-mass index in 200 countries from 1975 to 
2014: A pooled analysis of 1698 population-based measurement studies with 19.2 million participants. Lancet387, 1377-1396 (2016).

2. Halaas, J. L. et al. Weight-reducing effects of the plasma protein encoded by the obese gene. Science 269, 543-546 (1995).

3. Kamohara, S., Burcelin, R., Halaas, J. L., Friedman, J. M. \& Charron, M. J. Acute stimulation of glucose metabolism in mice by leptin treatment. Nature $389,374-377$ (1997).

4. Ahlma, R. S. et al. Role of leptin in the neuroendocrine response to fasting. Nature 382, 250-252 (1996).

5. Cowley, M. A. et al. Leptin activates anorexigenic POMC neurons through a neural network in the arcuate nucleus. Nature 411, 480-484 (2001).

6. Kristensen, P. et al. Hypothalamic CART is a new anorectic peptide regulated by leptin. Nature 393, 72-76 (1998).

7. Gropp, E. et al. Agouti-related peptide-expressing neurons are mandatory for feeding. Nat. Neurosci. 8, 1289-1291 (2005).

8. Wang, Q. et al. Interactions between leptin and hypothalamic neuropeptide $Y$ neurons in the control of food intake and energy homeostasis in the rat. Diabetes 46, 335-341 (1997).

9. Cohen, P. et al. Role for stearoyl-CoA desaturase-1 in leptin-mediated weight loss. Science 297, 240-243 (2002).

10. Levin, N., Nelson, C., Gurney, A., Vandleni, R. \& De Sauvage, F. Decreased food intake does not completely account for adiposity reduction after ob protein infusion. 
Proc. Natl. Acad. Sci. U. S. A. 93, 1726-1730 (1996).

11. Steinberg, G. R., Parolin, M. L., Heigenhauser, G. J. F. \& Dyck, D. J. Leptin increases FA oxidation in lean but not obese human skeletal muscle: Evidence of peripheral leptin resistance. Am. J. Physiol. - Endocrinol. Metab. 283, E187-E192 (2002).

12. Considine, R. V. et al. Serum immunoreactive-leptin concentrations in normal-weight and obese humans. N. Engl. J. Med. 334, 292-295 (1996).

13. Frederich, R. C. et al. Leptin levels reflect body lipid content in mice: Evidence for diet-induced resistance to leptin action. Nat. Med. 1, 1311-1314 (1995).

14. Liu, J., Lee, J., Hernandez, M. A. S., Mazitschek, R. \& Ozcan, U. Treatment of obesity with celastrol. Cel/161, 999-1011 (2015).

15. Feng, X. et al. IL1R1 is required for celastrol's leptin-sensitization and antiobesity effects. Nat. Med. 25, 575-582 (2019).

16. Lee, J. H. et al. Inhibition of NF-kB activation through targeting IkB kinase by celastrol, a quinone methide triterpenoid. Biochem. Phamacol. 72, 1311-1321 (2006).

17. Peng, B. et al. Mutations Y493G and K546D in human HSP90 disrupt binding of celastrol and reduce interaction with Cdc37. FEBS Open Bio 6, 729-734 (2016).

18. Yang, H., Landis-Piwowar, K., Chen, D., Milacic, V. \& Dou, Q. Natural Compounds with Proteasome Inhibitory Activity for Cancer Prevention and Treatment. Curr. Protein Pept. Sci. 9, 227-239 (2008).

19. Kyriakou, E. et al. Celastrol Promotes Weight Loss in Diet-Induced Obesity by Inhibiting the Protein Tyrosine Phosphatases PTP1B and TCPTP in the Hypothalamus. J. Med. Chem. 61, 11144-11157 (2018). 
20. Pfuhlmann, K. et al. Celastrol-induced weight loss is driven by hypophagia and independent from UCP1. Diabetes 67, 2456-2465 (2018).

21. Kim, J. Y., Hickner, R. C., Cortright, R. L., Dohm, G. L. \& Houmard, J. A. Lipid oxidation is reduced in obese human skeletal muscle. Am. J. Physiol. - Endocrinol. Metab. 279, E1039-E1044 (2000).

22. Goodpaster, B. H., Theriault, R., Watkins, S. C. \& Kelley, D. E. Intramuscular lipid content is increased in obesity and decreased by weight loss. Metabolism. 49, 467-472 (2000).

23. Goldsmith, R. et al. Effects of experimental weight perturbation on skeletal muscle work efficiency, fuel utilization, and biochemistry in human subjects. Am. J. Physiol. - Regul. Integr. Comp. Physiol. 298, R79-R88 (2010).

24. Dunaway, G. A. \& Kasten, T. P. Nature of the subunits of the 6-phosphofructo-1-kinase isoenzymes from rat tissues. Biochem. J. 242, 667-671 (1987).

25. Beard, D. A., Liang, S. D. \& Qian, H. Energy balance for analysis of complex metabolic networks. Biophys. J. 83, 79-86 (2002).

26. Vaisse, C. et al. Leptin activation of Stat3 in the hypothalamus of wild-type and ob/ob mice but not db/db mice. Nat. Genet. 14, 95-97 (1996).

27. Thyfault, J. P. et al. Impaired plasma fatty acid oxidation in extremely obese women. Am. J. Physiol. - Endocrinol. Metab. 287, 1076-1081 (2004).

28. Kloek, C. et al. Regulation of Jak kinases by intracellular leptin receptor sequences. J. Biol. Chem. 277, 41547-41555 (2002).

29. Zhang, J. et al. Oral bioavailability and gender-related pharmacokinetics of celastrol 
following administration of pure celastrol and its related tablets in rats. $J$.

Ethnopharmacol. 144, 195-200 (2012).

30. Wang, S., Liu, K., Wang, X., He, Q. \& Chen, X. Toxic effects of celastrol on embryonic development of zebrafish (Danio rerio). Drug Chem. Toxicol. 34, 61-65 (2011).

31. Zhu, H. et al. Synergistic anti-cancer activity by the combination of TRAIL/APO-2L and celastrol. Cancer Invest. 28, 23-32 (2010).

32. Kleinridders, A. et al. MyD88 Signaling in the CNS Is Required for Development of Fatty Acid-Induced Leptin Resistance and Diet-Induced Obesity. Cell Metab. 10, 249-259 (2009).

33. Zhang, X. et al. Hypothalamic IKKß/NF-KB and ER Stress Link Overnutrition to Energy Imbalance and Obesity. Cel/ 135, 61-73 (2008).

34. Ozcan, L. et al. Endoplasmic Reticulum Stress Plays a Central Role in Development of Leptin Resistance. Cell Metab. 9, 35-51 (2009).

35. Kaushik, S. et al. Autophagy in hypothalamic agrp neurons regulates food intake and energy balance. Cell Metab. 14, 173-183 (2011).

36. Muoio, D. M., Dohn, G. L., Fiedorek, F. T., Tapscott, E. B. \& Coleman, R. A. Leptin Directly Alters Lipid Partitioning in Skeletal Muscle. Diabetes 46, 1360-1363 (1997).

37. Minokoshi, Y. et al. Leptin stimulates fatty-acid oxidation by activating AMP-activated protein kinase. Nature 415, 339-343 (2002).

38. Obici, S., Feng, Z., Arduini, A., Conti, R. \& Rossetti, L. Inhibition of hypothalamic carnitine palmitoyltransferase-1 decreases food intake and glucose production. Nat. Med. 9, 756-761 (2003). 
39. Endo, T. A. et al. A new protein containing an $\mathrm{SH} 2$ domain that inhibits JAK kinases.

Nature 387, 921-924 (1997).

40. Masuhara, M. et al. Cloning and characterization of novel CIS family genes. Biochem.

Biophys. Res. Commun. 239, 439-446 (1997).

41. Plut, C., Ribière, C., Giudicelli, Y. \& Dausse, J. P. Hypothalamic Leptin Receptor and Signaling Molecule Expressions in Cafeteria Diet-Fed Rats. J. Pharmacol. Exp. Ther. 307, 544-549 (2003).

42. Ueki, K., Kondo, T. \& Kahn, C. R. Suppressor of Cytokine Signaling 1 (SOCS-1) and SOCS-3 Cause Insulin Resistance through Inhibition of Tyrosine Phosphorylation of Insulin Receptor Substrate Proteins by Discrete Mechanisms. Mol. Cell. Biol. 25,

8762-8762 (2005).

43. Bjørbaek, C., Elmquist, J. K., Daniel Frantz, † J, Shoelson, S. E. \& Flier, J. S. Identification of SOCS-3 as a Potential Mediator of Central Leptin Resistance. Mol. Cell $1,619-625$ (1998).

44. Kinjyo, I. et al. SOCS1/JAB is a negative regulator of LPS-induced macrophage activation. Immunity 17, 583-591 (2002).

45. Getty-Kaushik, L. et al. Mice deficient in phosphofructokinase-M have greatly decreased fat stores. Obesity 18, 434-440 (2010).

46. Zurlo, F., Larson, K., Bogardus, C. \& Ravussin, E. Skeletal muscle metabolism is a major determinant of resting energy expenditure. J. Clin. Invest. 86, 1423-1427 (1990). 


\section{Supplemental Figure legends}

Figure S1. 1-85 has equal leptin sensitization and weight losing effect to celastrol. Related to Figure 1.

(A-B) 1-85 had equal weight losing effect to celastrol in wild-type DIO mice. During 20 days treatment, both $1-85(0.15 \mathrm{mg} / \mathrm{kg}$ ) (from $43.07 \pm 3.60 \mathrm{~g}$ to $36.81 \pm 2.80 \mathrm{~g}, \mathrm{n}=7$ ) and celastrol $(0.15 \mathrm{mg} / \mathrm{kg}$ ) (from $42.97 \pm 2.27 \mathrm{~g}$ to $35.84 \pm 1.43 \mathrm{~g}, \mathrm{n}=7$ ) reduced body weight of DIO mice significantly, whereas the body weight of vehicle treated mice (from $43.85 \pm 3.75$ $\mathrm{g}$ to $40.73 \pm 3.87 \mathrm{~g}, \mathrm{n}=5$ ) showed no statistically significant decrease. (C) 1-85 had same leptin sensitization effect as celastrol. The eight weeks old wild-type lean mice $(n=5$ for each group) were treated with vehicle, celastrol $(0.15 \mathrm{mg} / \mathrm{kg})$ or $1-85(0.15 \mathrm{mg} / \mathrm{kg})$ for three days, and subsequently received either saline or leptin $(5 \mathrm{mg} / \mathrm{kg})$. The body weight was recorded. The body weight changes $24 \mathrm{hrs}$ after leptin administration were quantified. Vehicle+Saline, $-0.02 \pm 0.51 \mathrm{~g}$; Vehicle+Leptin, $-0.24 \pm 0.32 \mathrm{~g}$; Celastrol+Saline, $-0.48 \pm 0.26$ $\mathrm{g}$; Celastrol+Leptin, $-1.28 \pm 0.08 \mathrm{~g} ; 1-85+$ Saline, $0.40 \pm 0.49 \mathrm{~g}$; 1-85+Leptin, $-1.16 \pm 0.63 \mathrm{~g}$.

Figure S2. PFKM expression are successfully blocked by shRNA. Related to Figure 1.

Knockdown efficiency of Pfkm shRNA in L6 cells was examined by western blot.

Figure S3. The schematic diagram of Pfkm KO mice. Related to Figure 1.

(A) The schematic diagram of double-strand DNA donor generation. (B) Editing sites of

Crispr-cas9 system in Pfkm locus. (C) The critical exon 5/6 is flanked by two loxP sites in

Pfkm KO mice. (D) Relative Pfkm mRNA level of Pfkm KO mice and wild-type control in skeletal muscle measured by QPCR.

Figure S4. The serum leptin levels of DIO mice. Related to Figure 1 and 2.

(A) The serum leptin levels of Pfkm KO DIO mice $(21.00 \pm 21.87 \mathrm{ng} / \mathrm{mL}, \mathrm{n}=4)$ were significantly lower than wild-type DIO littermate $(71.94 \pm 13.60 \mathrm{ng} / \mathrm{mL})$. (B) The serum leptin levels of $A M P K \alpha 1 \alpha 2 \mathrm{mKO} D I O$ mice $(82.93 \pm 5.55 \mathrm{ng} / \mathrm{mL})$ were equal to wild-type DIO mice $(86.95 \pm 10.11 \mathrm{ng} / \mathrm{mL})$.

Figure S5. Loss of PFKM does not activate AMPK in both liver and hypothalamus. Related to Figure 1 and 2.

(A-B) Western blots show that both Pfkm KO and celastrol treatment does not affect phosphorylation of AMPK ${ }^{\text {Thr172 }}$ and ACC ${ }^{\text {Ser79 }}$, but induce phosphorylation on STAT3 ${ }^{\text {Tyr705 in }}$ both liver (A) and hypothalamus (B).

Figure S6. Phosphorylation on STAT3 $3^{\text {Tyr705 }}$ is induced by leptin in both central and periphery tissues. Related to Figure 2.

Wild-type lean mice at age of eight weeks old were subjected to acute intraperitoneal leptin administration $\left(5 \mathrm{mg} / \mathrm{kg}\right.$ ), and phosphorylation of STAT3 ${ }^{\text {Tyr705 }}$ were measure in both hypothalamus and skeletal muscles by western blot.

Figure S7. Celastrol reduce glycolytic rates in $293 \mathrm{t} / \mathrm{hLepRb}$ cells. Related to Figure 3.

(A) Celastrol inhibits PFKL at high concentration. The IC50 of celastrol was measured on 
recombinant PFKL. (B) Seahorse glycolysis stress test of $293 \mathrm{~T} / \mathrm{hLepRb}$ cells treated with celastrol.

Figure S8. SOCS1 are knocked down by shRNA. Related to Figure 3.

Knockdown efficiency of two SOCS1 shRNAs in 293T/hLepRb cells was examined by western blot against SOCS1.

Figure S9. 3-79 does not reduce body weight of regular lean mice. Related to Figure 4.

Body weight $(\mathrm{g})$ of eight-week old lean mice ( $\mathrm{n}=5$ for each groups) treated with vehicle or 3-79 $(1 \mathrm{mg} / \mathrm{kg})$ twice a day for 7 days.

Table S1. Protein list of Celastrol's target detected by ABPP in mice skeletal muscle. Related to Figure 1. Only the proteins with more than 1000 Mascot score in probe group but not in control group were picked.

Table S2. Targeted metabolimics analysis in celastrol treated L6 cells. Related to Figure 2.

Table S3. The levels of FFA in L6 cells. Related to Figure 2. The L6 cells were treated with $0 \mu \mathrm{M}, 2 \mu \mathrm{M}$ or $4 \mu \mathrm{M}$ celastrol for $30 \mathrm{~min}$.

Table S4. FFA levels in serum of DIO mice. Related to Figure 3. The DIO mice of wild type and $P f k m$ KO were subjected to daily intraperitoneal treatment of vehicle or 0.15 $\mathrm{mg} / \mathrm{kg}$ celastrol for 20 days.

Table S5. Structure and IC50 of original PFKM inhibitor candidates identified by High Throughput Screening. Related to Figure 4. 
Figure 1

$\underset{\text { Celastrol }}{\rightarrow}$

C

D
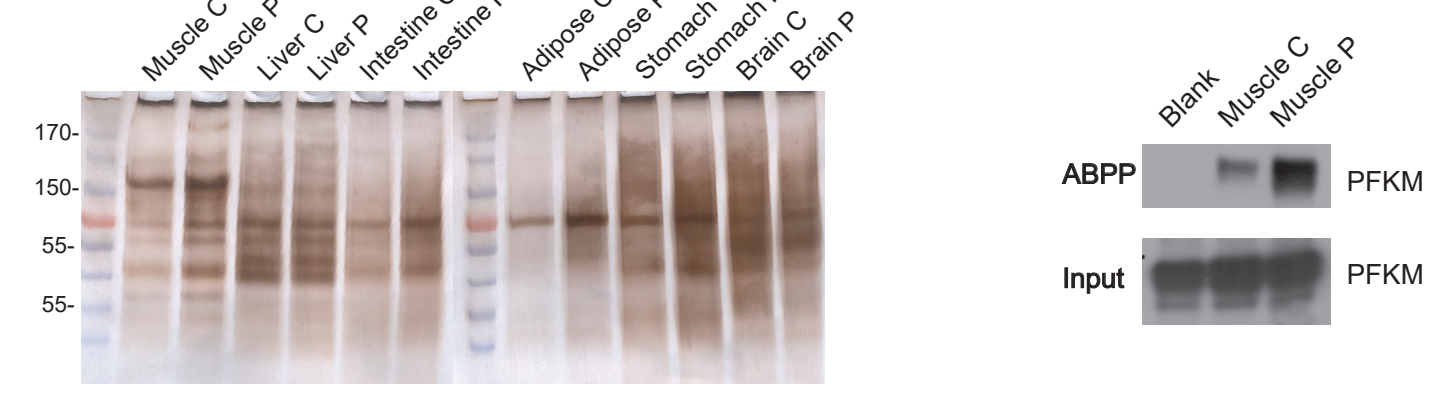

E
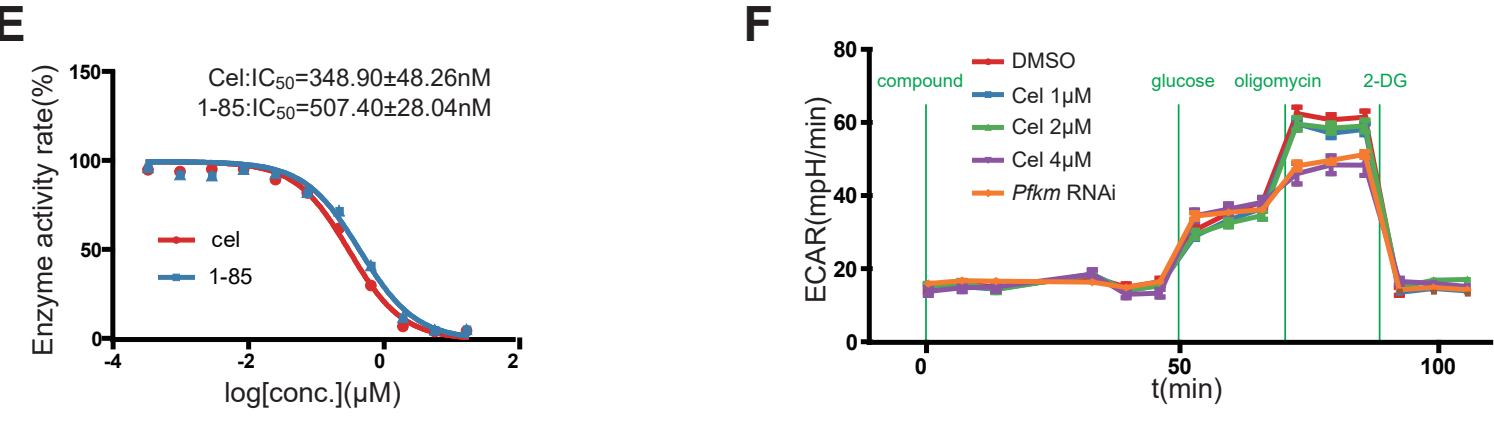

H

I Basic Glycolysis
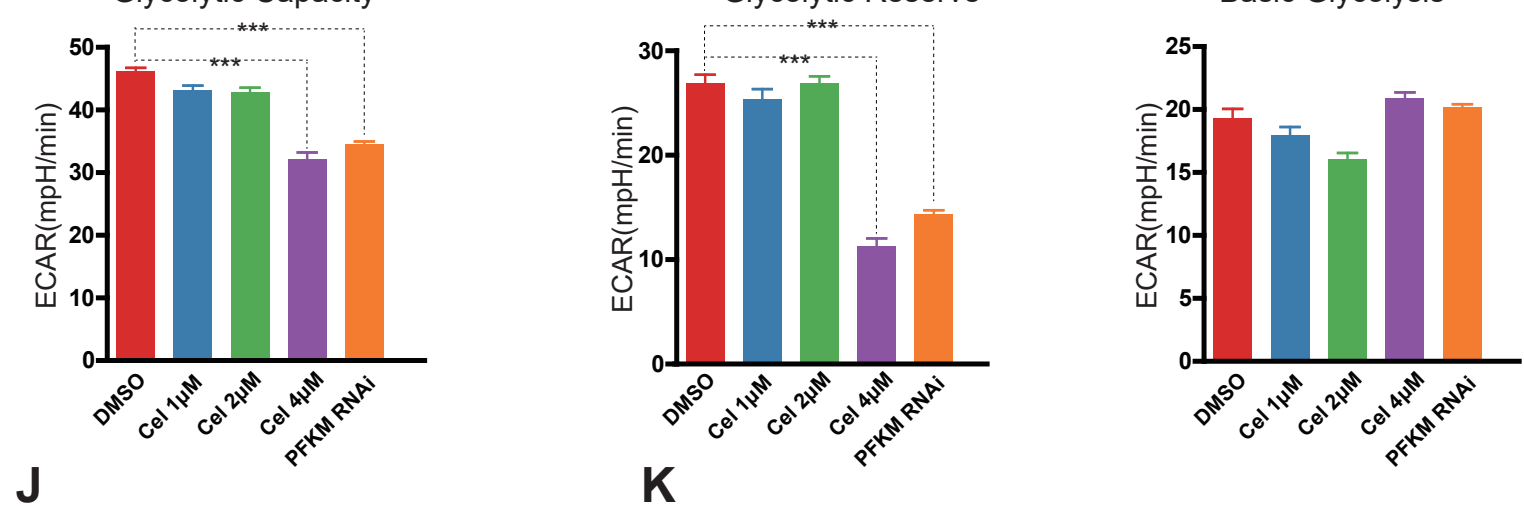

J $\mathrm{K}$
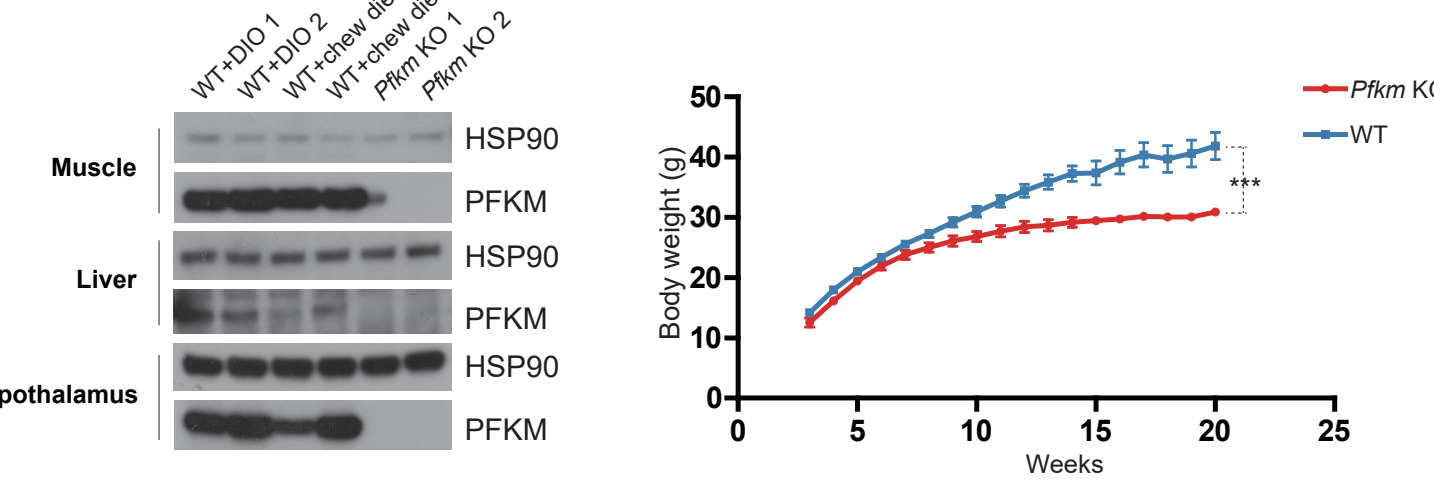

L

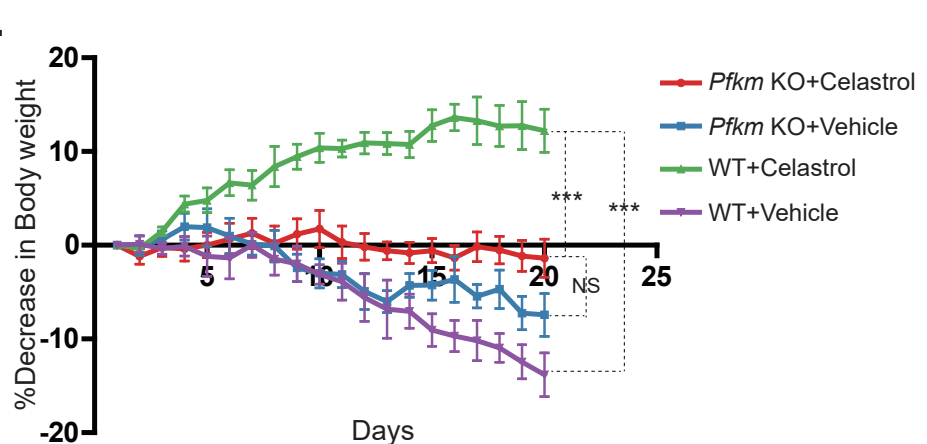

M

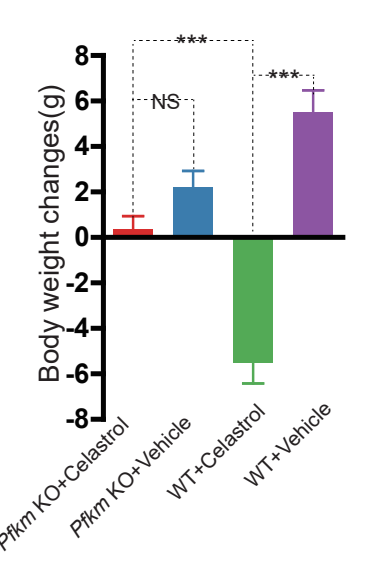

$\mathbf{N}$

0

Skeletal Muscle
Venicle Celastrol Venicle celastrol

Wild type Pikm KO

MSP90

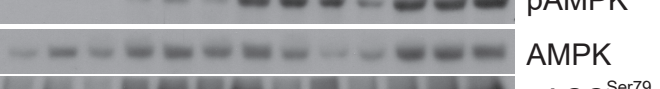

PACC

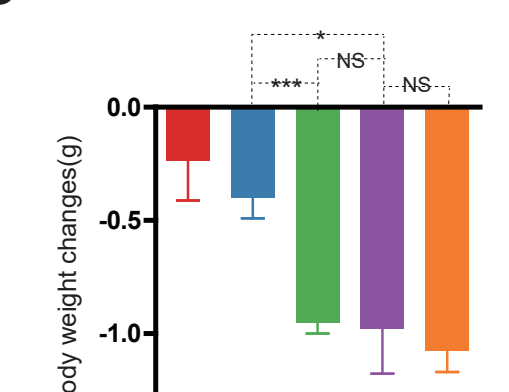

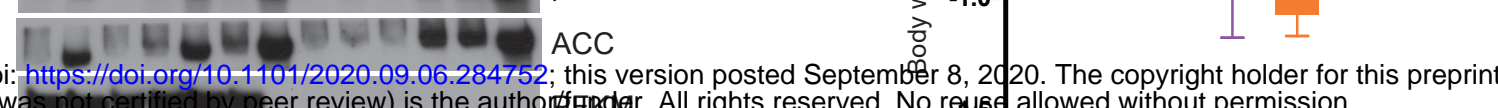

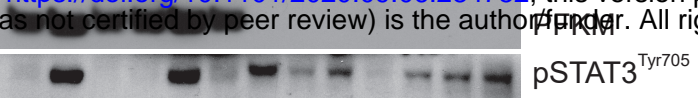


bioRxiv preprintgdidhttes://20i.org/10.1101/2020.09.06.284752; this version posted September 8, 2020. The copyright holder for this preprint (which was not certified by peer review) is the author/funder. All rights reserved. No reuse allowed without permission.

A
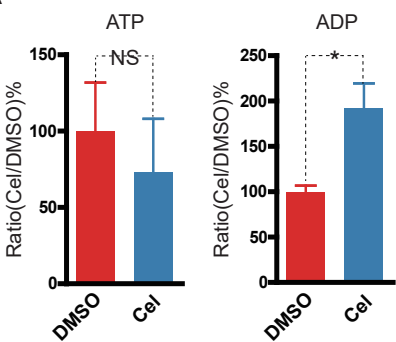

Malonyl-CoA

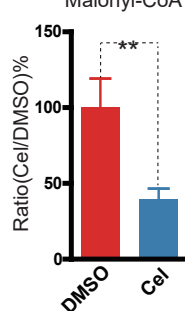

$B$

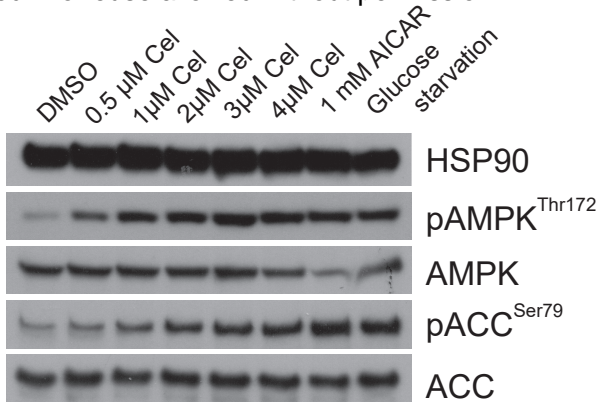

C

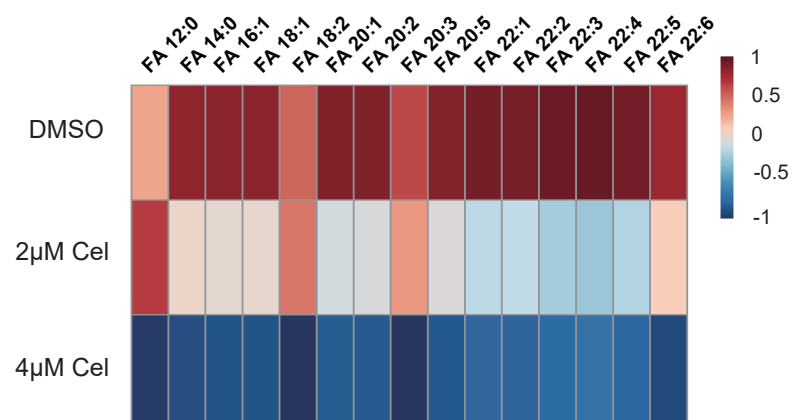

$\mathbf{E}$

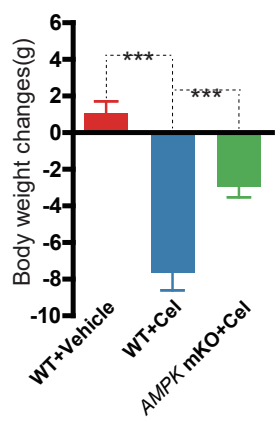

G

Liver

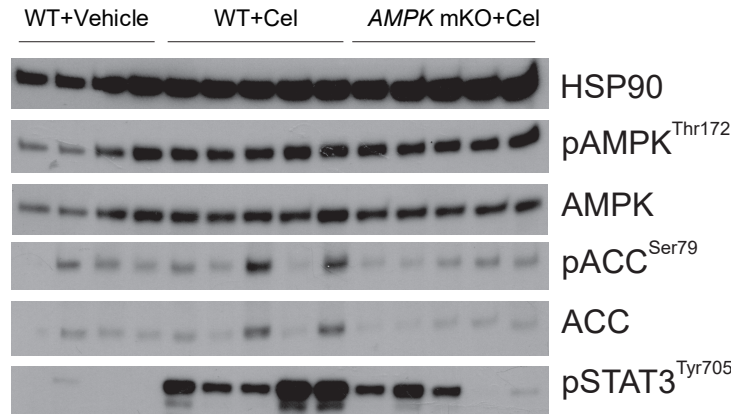

F Skeletal Muscle

H

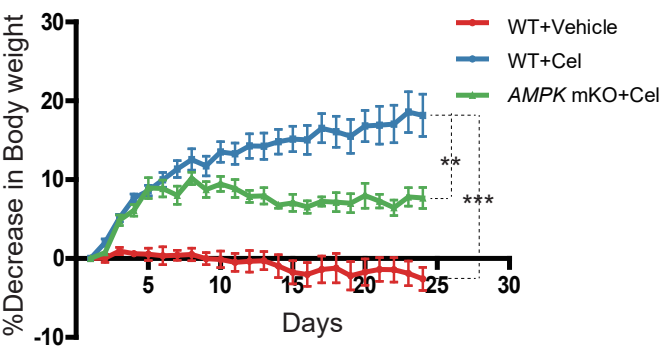

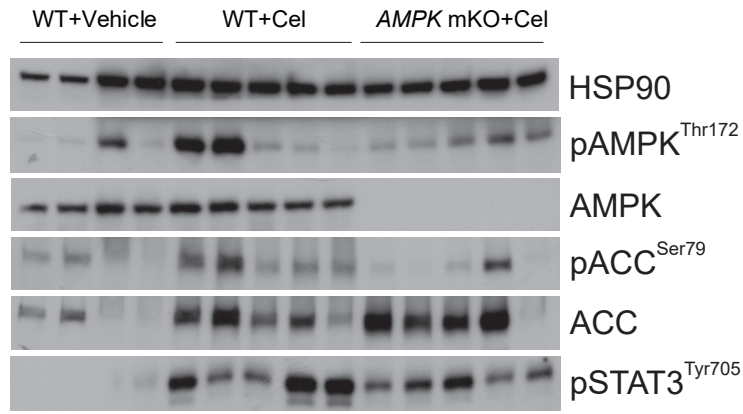

Hypothalamus

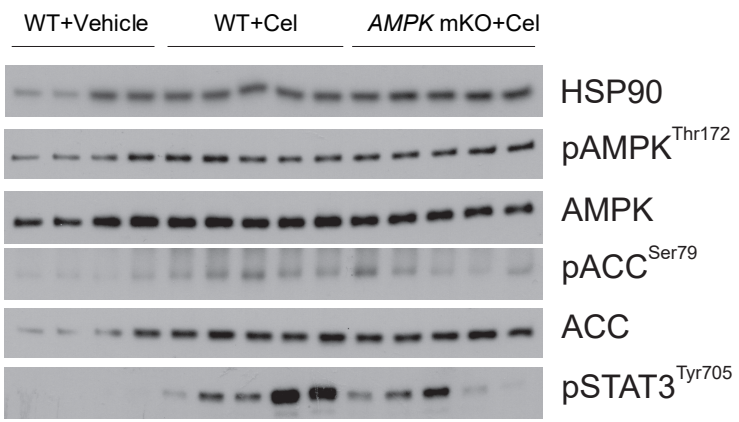




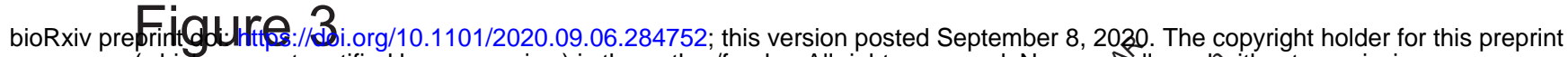

(which was not certified by peer review) is the author/funder. All rights reserved. No reugetllowegewithout permission.

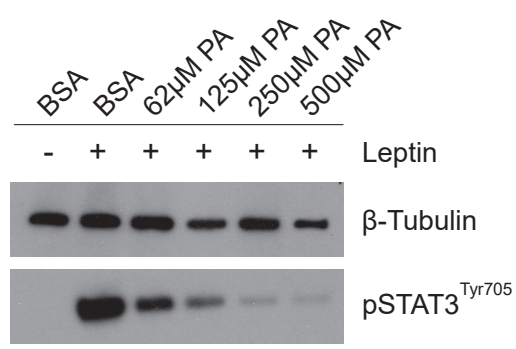

C

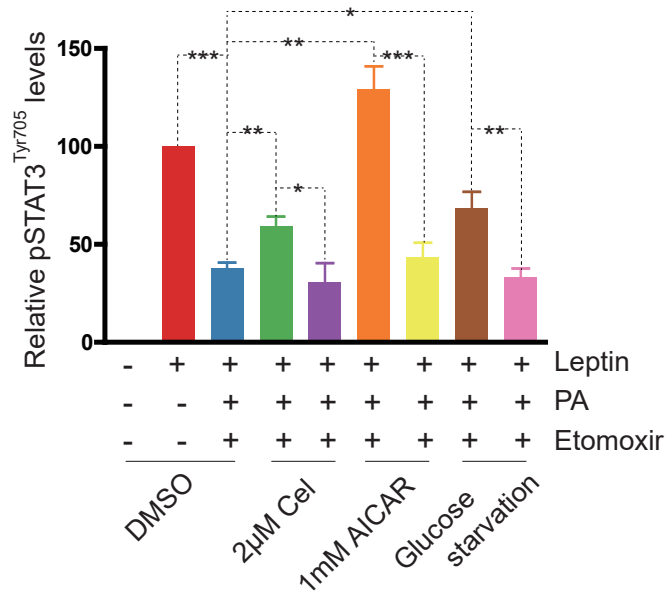

E

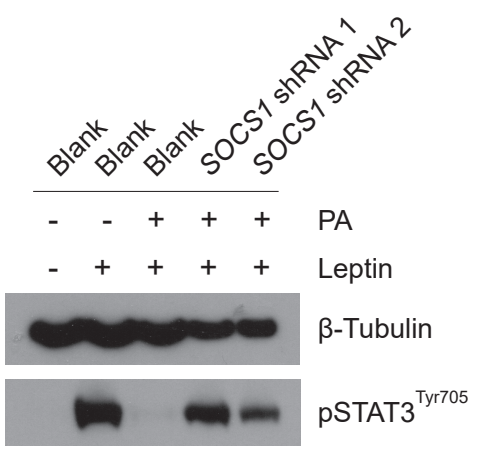

F

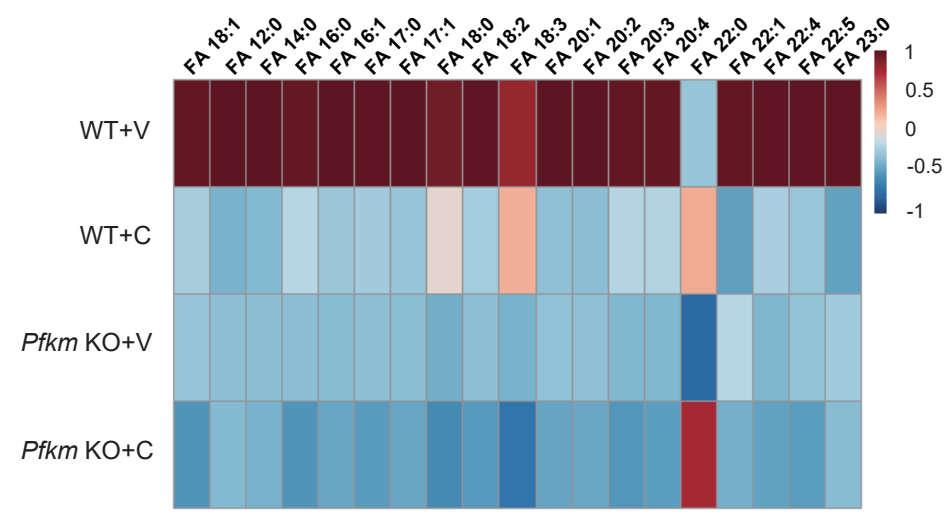

D
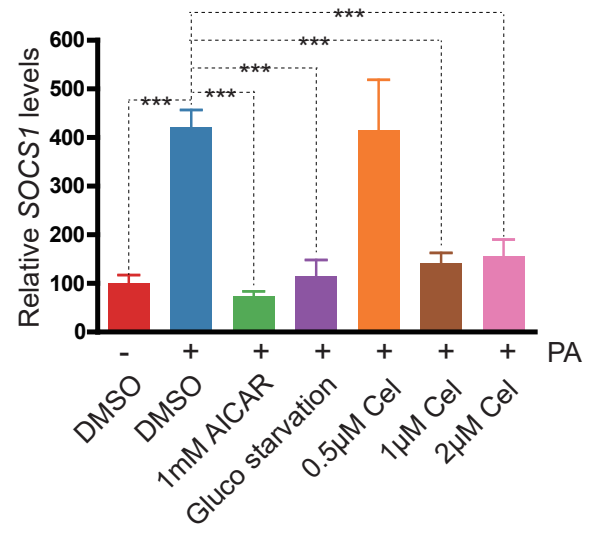

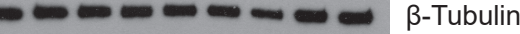

$=-\infty-\infty$ pSTAT3 $^{\text {Tyr705 }}$

$\mathbf{G}$

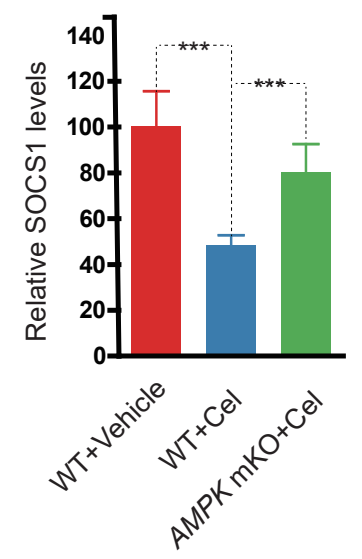


bioRxiv preFridtgdillttes:/4tbi.org/10.1101/2020.09.06.284752; this version posted September 8, 2020. The copyright holder for this preprint (which was not certified by peer review) is the author/funder. All rights reserved. No reuse allowed without permission.

A<smiles>O=C(c1cccs1)c1no[n+]([O-])c1C(=O)c1cccs1</smiles>

3-79

D Glycolytic Capacity

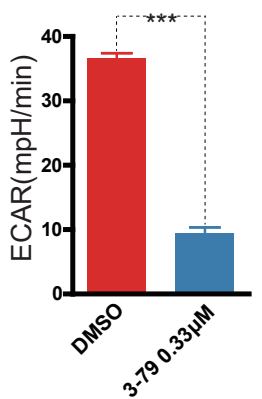

H

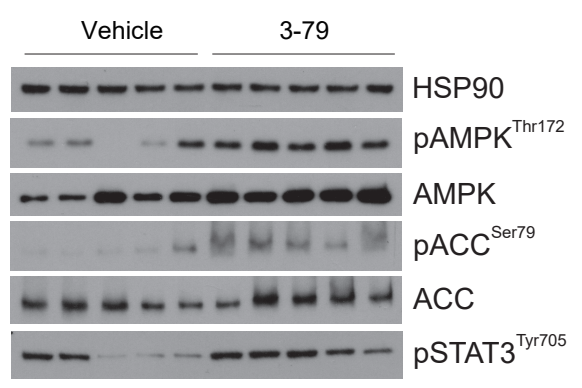

B

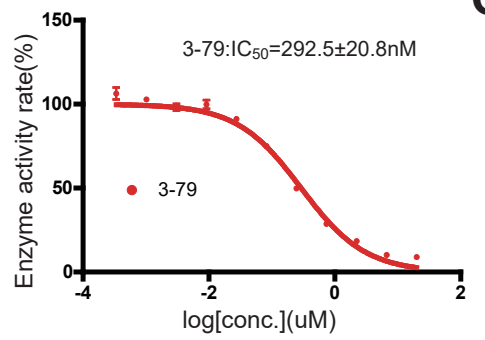

$\mathbf{F}$

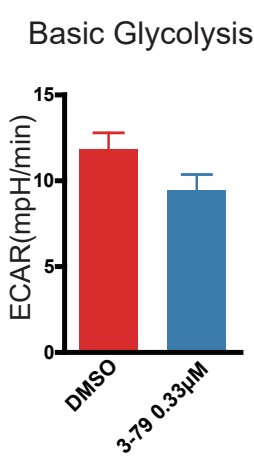

C

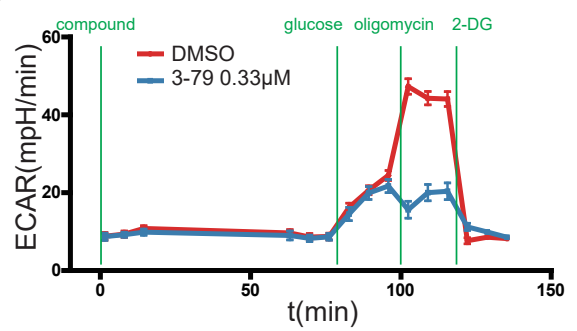

G
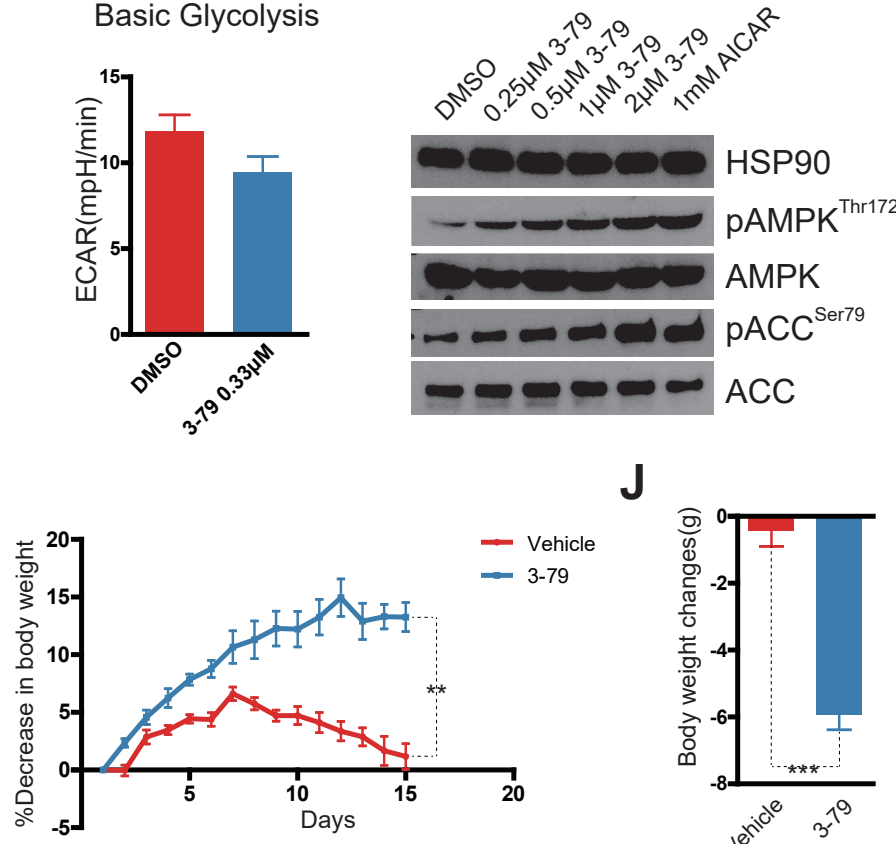

$\mathbf{J}$

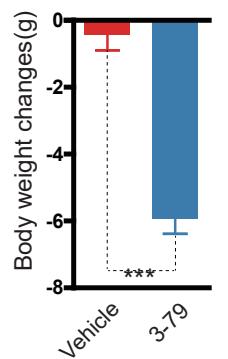

